\title{
Opportunity recognition for small businesses in medical tourism in Taiwan
}

\author{
Ying-Hsun Hung, Jerome Chih-Lung Chou, Jung Ma, and Ching-pei Lin ${ }^{a}$ \\ Department of Information Management, Hwa-Hsia Institute of Technology, Taiwan, ROC.
}

\begin{abstract}
Medical tourism has become an investment focus for many Asian countries. For example, India, Singapore, Thailand, South Korea, and Malaysia have governmental projects to foster this emerging industry, and successfully attract millions of medical tourists each year. Medical tourism consists of diverse industries that can provide abundant opportunities of new businesses. Currently Taiwan is trying to catch up in this trend by making national development policies, setting up institutions, modifying laws and regulations, and encouraging investment of private sector. The purpose of this study is to analyze the status quo of international medical tourism in Taiwan, and identify several opportunities for small businesses to catch and to participate in forming a solid medical tourism industry for Taiwan.
\end{abstract}

\section{Introduction}

Medical tourism has materialized from a broader concept of health tourism as one's organized travel outside their local environment for the maintenance, enhancement, or restoration of one's well-being in mind and body. Particularly, in medical tourism the travelers seek medical intervention such as cosmetic enhancements, dental treatments, or major or minor required surgery. Medical tourism is partly resulted from globalization of healthcare and tourism itself constituting huge economic potential (Bookman \& Bookman, 2007).

Medical tourism is also a popular cultural phenomenon when people make a long journey in order to obtain medical services while vacationing (Connell, 2006). Medical travelers are looking for specialty treatments, privacy, expedited medical attention, or, frequently, lower costs than available at home country (Woodman, 2008). The market drivers of medical tourism are cost savings, comparable or better quality, and quicker access to the care (Keckley, 2008). Medical or health tourism has become one of the fastest growing sectors that many countries are embracing for planning their economic expansion because it drives the development of a high-value and technology oriented service industry. The growth of medical tourism has been reportedly high for each year recently.

\section{Medical tourism in Taiwan}

Taiwan has full spectrums of medical services, including preventive medicine, health screening, diagnosis, and treatment of disease. All services are accompanied with advanced equipment and customized health examination, or more diversified herbal medicine and diagnosis. The five

\footnotetext{
${ }^{\mathrm{a}}$ Corresponding author : jerome@cc.hwh.edu.tw

(C) The Authors, published by EDP Sciences. This is an open access article distributed under the terms of the Creative Commons Attribution License 4.0 (http://creativecommons.org/licenses/by/4.0/).
} 
competitive items of medical tourism of Taiwan are craniofacial reconstructive surgery, living donor liver transportation, artificial reproductive technology, cardiovascular therapy, and joint replacement surgery $(\mathrm{Wu}, 2009)$. Price competitive items in the international markets of Taiwan are health examination, surgery of prostate, hysterectomy and face lifts.

Taiwanese physicians must finish 7 years of medicine school education: 4 years of basic medicine courses, 2 years of specialized medicine courses, and 1 year of internship training. Besides, they must be certified by a national examination. A qualified doctor-in-charge must go through extra years of specialist training, do research and publish proper papers. Averagely, it takes 12-13 years for a student of school of medicine to become a doctor-in-charge. The high quality of medicine school education and practical training enable Taiwan to provide high quality of medical services.

Currently, there exist several referral organizations helping recruit medical travelers to Taiwan, such as Zion Health Control and Youth Rejuvenation Center, Formosa Medical Travel, and World Taiwanese Chambers of Commerce.

However, the development of medical tourism of Taiwan is facing some problems that limit its potential. Firstly, the distribution of nationality of medical travelers to Taiwan is largely skewed to mainland China. Due to recent improvement of political relationship with China, the number of Chinese tourist to Taiwan has been increasing, and some of them went to Taiwan with medical visas in tours. These tours are recruited by travel agents of any kind in China, and the medical visa is issued easily with the help of 39 selected Taiwanese hospitals. Because the demand of tourism to Taiwan is high in China and there exists quota for Chinese travelers to Taiwan, medical tours became one of the special channels for Chinese travelers whose need are not really medical.

Secondly, the distribution of medical services consumed is skewed to low priced items. Since many of travelers with medical visas go to Taiwan on purposes other than obtaining medical treatments, they tend to spend less in hospital. Recently, Taiwanese government posed a new regulation on the minimum requirement on medical expenses of travelers who apply medical visa through hospitals. The new regulation mandates hospitals that accommodate medical travelers must present receipt exceeding 10,000 NTD (about 340 USD) in advance when applying medical visa for the patient.

Thirdly, famous and talented Taiwanese physicians are attracted to mainland China to provide high-end medical services. Chinese hospitals offer advanced equipment, convenient and condensed schedule, and high payments to Taiwanese physicians in fields like anti-aging, dental implant and cosmetic surgery, which are not covered by Taiwan National Health Insurance. Such arrangement is good for physician's private benefit, but does not help the development of medical tourism industry in Taiwan.

\section{Issues of incentive and information}

The problems above root in incentive and information gaps among the immature industry. Because of the broad coverage of National Health Insurance system of Taiwan, part of the role of large hospitals in Taiwan is social welfare provider. Large hospitals receive large government subsidy and serve large amount of citizen patients. This leaves little capacity and poor incentive for large hospitals to serve international patients as long as the income from National Health Insurance is big. However, famous physicians and advanced equipment resides in large hospitals. Recently, due to financial shortage of National Health Insurance, some large hospitals has stared rearrange organization and capacity for more international patients.

Traditional travel agents have incentive problems, too. Due to the difficulty to serve diverse medical needs of travelers, serving clients of medical tourism are not easy to provide high profit margin to travel agency. Therefore travel agents lack of incentive to promote medical tourism through their cooperative overseas partners, which is the most effective channel to disperse tourism information overseas, and consequently, the information about the benefit and advantage of Taiwan medical services are poorly communicated over this traditional channel. 
Local hotels and local health promotion service providers such as hot spring resort, oxygen bar, functional food store and medical equipment venders are well motivated to welcome international medical travelers, but they are not proper to take the leading role to coordinate the joint effort of international marketing and medical service providing.

\section{Role of medical travel facilitator}

To solve the incentive and information problems, fostering the development of medical travel facilitator is one solution. Growth of medical tourism has been facilitated by the emergence of healthcare intermediaries or medical tourism facilitators between international patients and hospital networks (Connell, 2006). Medical travelers could seek help from medical travel facilitators in finding reliable healthcare providers, ensuring trouble-free travel arrangements and having a comprehensive understanding of any pre and post operation cautions. Facilitator could close the incentive and information gaps by integrating knowledge of medical services, health services, tourism facilitation and concierge services, which are currently poorly performed by hospitals, traditional travel agents, and local hotels.

Services provided by medical travel facilitators can be stand alone or in a form of partnership integration. Partnering with industry key players can provide better start-to-finish experience to travelers and thus create more value for the industry. These services can include air travel and visa arrangement, long-distance medical screening and consultation, communication between physicians of parent and host countries, transportation of medical records, arrangement of personal nurse, arrangement of accommodation, and arrangement of follow-up care.

\section{Opportunities for small business}

Applying TRIZ theory, we identify several opportunities that are suitable for small and medium enterprises (SME) to catch.

To close the incentive gap of large hospitals in serving international patients, SMEs with medical technology background could act as overseas liaison for large hospitals if they have overseas subsidiaries or strong online presence. As a liaison, the SME could access the service capacity information of hospital and only use the hospital allocated medical service items and capacity to help foreign medical travelers plan their journeys to Taiwan. With the endorsement of large hospitals from the parent country, the SME could improve its brand image and enhance profitability. With liaisons, large hospitals could close the gap of information about high-end and advantageous medical services in the global market. The candidate SMEs for hospital liaisons are medicine, functional food and medical equipment providers, many of which could be carefully selected from the hospital's current suppliers.

To close the incentive gap of traditional travel agents, SMEs with staff of local tourist guides could act as outside suppliers of medical concierge services of travel agents. Traditional travel agents could advertise their allying hospital networks with medical concierge services to their clients of free and independent travel (FIT). And as the service is included in the FIT package, the SMEs could expect more quantity of genuine medical travelers and provide them customized local services with high value, such as translation and personal nursing care, in coordination with local hospitals, hotels and health promotion service providers.

\section{Methodology}

Medical tourism consists of diverse industries that can provide abundant opportunities of new businesses. However, few studies in tourism and medical cares have discussed the medical tourism services, or proposed the services quality evaluation strategies to assist in improving performance for long-term development. The purpose of this study is to address this problem using the novel method 
of hybrid MCDM (multiple criteria decision-making), including DEMATEL (decision-making trial and evaluation laboratory), DANP (the DEMATEL-based analytic network process) and VIKOR, to examine the influential relationships among dimensions and criteria of the empirical case and to ultimately present the best improvement schemes, which are valuable for both practitioners and researchers.

The decision-making process involves identifying problems, constructing preferences, evaluating alternatives, and determining the best alternative. However, when decision-makers evaluate the alternatives with multiple criteria, many problems, such as the weights of the criteria, preference dependence, and conflicts among criteria, seem to complicate the decision making process and should be resolved by more sophisticated methods. The DEMATEL (Decision MAking Trial and Evaluation Laboratory) method gathers collective knowledge to capture the causal relationships between strategic criteria. This paper applies the DEMATEL method in the strategic planning of medical services Gaps to help managers address the above situations and related questions. The procedures of the DEMATEL method and the evaluation methods can be summarized as follows:

Step 1: Producing the direct-relation matrix

Comparison scale of the DEMATEL method

\begin{tabular}{cl}
\hline Numeral & Definition \\
\hline 0 & No influence \\
1 & Low influence \\
2 & Medium \\
3 & High influence \\
4 & Very high influence \\
\hline
\end{tabular}

Step 2: Normalizing the direct-relation matrix

On the basis of the direct-relation matrix $\boldsymbol{A}$, the normalized direct-relation matrix $\boldsymbol{X}$ can be obtained through formulas (3-1) and (3-2):

$$
\begin{gathered}
\boldsymbol{X}=k \cdot \boldsymbol{A} \\
\max _{1 \leq i \leq n} \sum_{j=1}^{n} a_{i j} \\
i, j=1,2, \ldots, n
\end{gathered}
$$

Step 3: obtaining the total-relation matrix

Once the normalized direct-relation matrix $\boldsymbol{X}$ has been obtained, the total-relation matrix $\boldsymbol{T}$ can be derived by using formula (3-3), in which the $\boldsymbol{I}$ denotes the identity matrix.

$$
\begin{aligned}
& \boldsymbol{T}=\boldsymbol{X}+\boldsymbol{X}^{2}+\boldsymbol{X}^{3}+\ldots+\boldsymbol{X}^{q}=\boldsymbol{X}\left(\boldsymbol{I}+\boldsymbol{X}+\boldsymbol{X}^{2}+\ldots+\boldsymbol{X}^{q-1}\right) \\
& =\boldsymbol{X}\left(\boldsymbol{I}+\boldsymbol{X}+\boldsymbol{X}^{2}+\ldots+\boldsymbol{X}^{q-1}\right)(\boldsymbol{I}-\boldsymbol{X})(\boldsymbol{I}-\boldsymbol{X})^{-1} \\
& =\boldsymbol{X}\left(\frac{\boldsymbol{I}-\boldsymbol{X}^{n}}{I-\boldsymbol{X}}\right) \\
& =\boldsymbol{X}(\boldsymbol{I}-\boldsymbol{X})^{-1}{ }_{\text {, when }}{ }_{q \rightarrow \infty, \boldsymbol{X}^{q}=0} \\
& \boldsymbol{T}=\boldsymbol{X}(\boldsymbol{I}-\boldsymbol{X})^{-1}
\end{aligned}
$$

Step 4: Analyzing the results

In this step, the sum of rows and the sum of columns are used to derive vector $\boldsymbol{S}$ and vector $\boldsymbol{r}$ respectively, as shown in formulas (3-3), (3-4), and (3-5). Then, the horizontal axis vector $(\boldsymbol{S}+\boldsymbol{r}$ ), called "Prominence", is formed by adding $\boldsymbol{S}$ to $\boldsymbol{r}$, which indicates the level of importance of the criterion. Similarly, the vertical axis ( $\boldsymbol{S}-\boldsymbol{r})$ called "Relation", is formed by 
subtracting $\boldsymbol{S}$ from $\boldsymbol{r}$, which may divide criteria into a cause group and an effect group. When $(\boldsymbol{S}-\boldsymbol{r})$ is positive, the criterion belongs to the cause group; otherwise, it belongs to the effect group. Therefore, the causal diagram can be derived by mapping the dataset of $(\boldsymbol{s}+\boldsymbol{r}, \boldsymbol{s}-\boldsymbol{r})$, which provides valuable insights for making decisions.

$$
\begin{gathered}
\boldsymbol{T}=\left[t_{i j}\right]_{n \times n, i, j=1,2, \ldots, n} \\
\boldsymbol{s}=\left[\sum_{j=1}^{n} t_{i j}\right]_{n \times 1}=\left[t_{i}\right]_{n \times 1} \\
\boldsymbol{r}=\left[\sum_{i=1}^{n} t_{i j}\right]_{1 \times n}^{t}=\left[t_{\cdot j}\right]_{n \times 1}
\end{gathered}
$$

where vector $\boldsymbol{S}$ and vector $\boldsymbol{r}$ respectively denote the sum of rows and the sum of columns from the total-relation matrix $\boldsymbol{T}=\left[t_{i j}\right]_{n \times n}$.

Opricovic (1998) and Opricovic and Tzeng (2002) developed VIKOR (the Serbian name, VlseKriterijumska Optimizacija I Kompromisno Resenje, means Multi-criteria Optimization and Compromise Solution). The basic concept of VIKOR lies in defining the positive and negative ideal solutions first. The positive ideal solution indicates the alternative with the highest value (score of 100), while the negative ideal solution indicates the alternative with the lowest value (score of 0 ). In our study, the highest performance value of medical services is 5, and the lowest performance value is 0 . They were used to help DMs to represent the present status of services components for services assessment and adoption.

The VIKOR method was developed as a multi-criteria decision-making method to solve a discrete decision problem with non-commensurable and conflicting criteria (Opricovic and Tzeng 2004). The method ranks a set of alternatives, and selects the alternative with the highest score. It then suggests compromise solutions to a problem with conflicting criteria in order to help practitioners reach a final decision. Here, the compromise solution is the feasible solution that is the closest to the ideal, and a compromise means an agreement reached on the basis of mutual concessions.

\section{Conclusion}

Medical tourism sits at the growing intersections of medical technology, economy, cultural and global relations. However, Taiwan is sitting at the edge of market failure due to incentive and information gaps among key industry players. Medical travel facilitator could help close these gaps, and small businesses can find opportunities and play the facilitator role in shaping a healthy medical tourism industry for Taiwan. This study applies a modified VIKOR method to improve service quality among Medical Tourism Services in Taiwan. Our model allows decision-makers and policy-makers to understand the gaps between alternatives and aspired-levels in practice. We then applied the modified VIKOR method to establish the gaps in priorities between alternatives and aspired-levels. Finally, based on these gaps in priorities, we provide managerial implications to improve different carriers for satisfying the medical tourism customers' needs to achieve the aspired-level.

\section{References}

1. M.Z. Bookman, K.R. Bookman, Medical tourism in developing countries (NY: Palgrave Macmillan, 2007)

2. J. Connell, Tourism Management, 27(6), 1093-1100 (2006) 
3. P.H. Keckly, Medical tourism: Consumers in search of value. Washington DC: Deloitte Center for Health Solutions (2008)

4. J. Woodman, Patients beyond borders: Everybody's guide to affordable, world-class medical tourism. Chapel Hill, NC: Healthy Travel Media (2008)

5. H. Wu, Database of Industry \& Technology Intelligence Service: Current status of medical tourism in Taiwan., Ministry of Economic Affairs of Taiwan (2009)

6. Y.H. Hung, T.L. Huang, J.C. Hsieh, H.J. Tsuei, C.C. Cheng and G.H. Tzeng, Knowledge-Based Systems, 35, 87-93 (2012)

7. A. Pourahmad, A. Hosseini, A .Banaitis, H. Nasiri, N. Banaitienè and GH Tzeng, Technological and Economic Development of Economy, 21(5), 773-796 (2015)

8. K.Y. Shen, G.H. Tzeng, Soft Computing, 19(4), 859-874 (2015) 